\title{
Aproximación al discurso referido en el habla infantil ${ }^{1}$
}

\author{
María José Gallucci - Universidad Central de Venezuela / Universidad de Zaragoza \\ gallucci@unizar.es \\ Yanira Pinto - Universidad Central de Venezuela \\ yaniracpintoh@gmail.com
}

Rebut / Received: 15-11-15

Acceptat / Accepted: 29-2-16

Resum. Aproximació al discurs referit en la parla infantil. En aquest treball estudiem l'ús del discurs referit en la parla infantil. Descrivim l'estil directe, l'estil indirecte i les cites onomatopeiques des d'una perspectiva sintacticopragmàtica en una mostra de 28 parlants pertanyents al Corpus de habla infantil (Shiro, 1996) tenint en compte el tipus de cita, els marcs introductors, els verbs més freqüents que emmarquen les cites i l'atribució de la paraula. També indaguem, en funció del tipus de l'entrevista analitzada i de les tasques que van dur a terme els nens, quin tipus de fet citen en les seves narracions, és a dir, si són reals o ficticis. Els resultats generals obtinguts mostren que els parlants s'inclinen per l'ús de l'estil directe, sobretot amb el verb dir com a marc introductor, prefereixen citar els altres més que a si mateixos o el propi grup i utilitzen el discurs referit especialment en les narracions fictícies.

Paraules clau: discurs referit, parla infantil, espanyol de Caracas.

Abstract. On reported speech in child discourse. The present study investigates reported speech in child discourse. Quotes in direct and indirect styles, and onomatopoeias are analyzed from a syntactic and pragmatic perspective using a sample of 28 speakers of the Corpus de habla infantil (Shiro, 1996). The analysis focuses on the following aspects: type of quotation, frame, frame verbs and speech attribution. In addition, the study examines whether children refer to

\footnotetext{
1. Una parte de este artículo fue elaborada durante una estancia de investigación doctoral financiada por el Banco Santander y la Universidad de Zaragoza. Agradecemos a los evaluadores de la revista los comentarios y las sugerencias que han enriquecido el texto que aquí presentamos.
} 
real or fictional events in their narrations. Results show that a) direct style is the most used type of discourse, especially with the verb decir as a grammatical form that introduces quotes, b) speakers prefer to quote somebody else's words and, c) children mostly use reported speech to give accounts of fictional events.

Keywords: reported speech, child discourse, Spanish spoken in Caracas.

\section{Reflexividad del lenguaje y discurso referido}

Una de las diferencias fundamentales entre el ser humano y otras especies es el desarrollo de un sistema de signos lingüísticos que diferencia la comunicación humana del lenguaje animal: la lengua. En el marco de este sistema, los hablantes emplean distintos mecanismos para reproducir palabras gracias a la función metalingüística del lenguaje (Jakobson, 1975). Esta función, ampliamente relacionada con la denominada reflexividad del lenguaje (Lucy, 1993), junto con la doble articulación (Martinet, 1972) y la prevaricación, individualiza a las lenguas naturales frente a otros sistemas comunicativos (Hockett, 1958). ${ }^{2}$ Se trata de una herramienta discursiva empleada para expresar lo que alguien ha dicho —o dirá- en un momento dado y que incluso puede funcionar como mecanismo de legitimación y argumentación. No estamos ante una mera repetición de palabras sino, más bien, frente a un recurso "consciente, reformulador y (re)creativo" (Marcuschi, 1997, p. 188) que implica mucha imaginación y, en algunos casos, gran histrionismo.

La reflexividad suele estar presente en todo tipo de textos, bien sean orales o escritos, pero, especialmente, en los relatos cotidianos. El interés por estudiar la reflexividad lingüística y, en especial, la incorporación de otras voces en el discurso - aunque no propiamente aunada a la oralidad-surge en los ańos setenta, a pesar de que ya medio siglo antes años Bajtín había dado algunas pistas al respecto. Los aportes más conocidos sobre este particular corresponden al dialogismo propuesto por el propio Bajtín (1981, 1982), a la teoría polifónica de la enunciación de Ducrot (1986), y a la heterogeneidad mostrada planteada por Authier-Revuz (1984, 1995, 1996). Una de las manifestaciones de la reflexividad y de estas teorías es el discurso referido.

Reyes (1995) explica que el discurso referido (en adelante, DR) pone en contacto un texto presente con otro anterior (que a su vez puede contener otros más) y, en este sentido, el texto funciona como cajas chinas o muñecas rusas. Con esta metáfora, la

2. Como es bien sabido, la doble articulación planteada por Martinet (1972) propone que toda lengua está estructurada en dos niveles: i) unidades mínimas carentes de significado (fonemas), y ii) unidades mínimas dotadas de significado (morfemas, unidades léxicas). Por su parte, la prevaricación consiste en la posibilidad de emitir mensajes que no sean verdaderos. Aunque Hockett (1958) se refiere a la primera como dualidad, ambos aspectos forman parte de las propiedades del lenguaje humano descritas por el autor y se han empleado tradicionalmente para diferenciar la comunicación humana del lenguaje animal. 
autora ilustra lo increíblemente flexible que es la lengua y, a su vez, lo prodigiosa que es la mente humana para descifrar estos intrincados vericuetos lingüísticos.

El DR consiste entonces en la "puesta en relación de discursos donde uno de ellos crea un espacio enunciativo particular, mientras que el otro es colocado a distancia y atribuido a otra fuente, de manera unívoca o no" (Rosier, 1999, p. 125). Charaudeau y Maingueneau (2005, p. 184) destacan la problemática que surge a propósito de los diversos modos en los que se representan en el discurso palabras atribuidas a "instancias distintas del locutor".

A pesar de que el DR comprende diversos mecanismos de citación, en este trabajo nos centraremos únicamente en el estilo directo (ED), en el estilo indirecto (EI) y en las denominadas citas onomatopéyicas $(\mathrm{CO})$, tres de esos procedimientos citativos a través de los cuales se materializa explícitamente ${ }^{3}$ el DR.

Bolívar (1998-1999) explica que el ED implica citar sin cambiar el centro deíctico ni las palabras del enunciado original; sin embargo, este último aspecto es relativo pues, aunque exista la voluntad del hablante de expresar literalmente la información que se transmite, no siempre ocurre de esta manera. Compartimos la opinión de Reyes (1995, pp. 7-8) sobre este particular:

Mediante la cita, un hablante atribuye a otro ciertas palabras: ya sea las palabras exactas, ya sea su contenido, ya sea una mezcla variable de ambas cosas. Estas palabras quedan, así, atribuidas: citar es siempre atribuir intencionalmente. La atribución puede ser falsa (si hacemos decir a una persona algo que nunca dijo o que nunca dijo de esa manera), puede ser aproximativa (cuando no citamos literalmente, sino resumiendo o reconstruyendo el discurso del otro), o puede ser ficticia (como las palabras de los personajes literarios).

En (1) ofrecemos un ejemplo de ED tomado de la muestra analizada.

(1) *CHI: le dijo [c] <muchacha, hágame el favor [c], y cómpreme una malta para la niña [c] que está enferma> [“] [c] $(\mathrm{ED})^{4}(\text { Leonmailín })^{5}$

3. Usamos el adverbio explícitamente para referirnos a la heterogeneidad mostrada planteada por Authier-Revuz (1982), es decir, a aquella que corresponde a la presencia localizable de otros discursos en una enunciación dada. Por su parte, la heterogeneidad constitutiva tiene que ver con la presencia del interdiscurso en todo texto. En este último caso, no se trata solamente de un espacio en el que vendría a introducirse desde fuera el discurso de otro, sino que se constituye, y esta es la palabra clave, "a través de un debate con la alteridad, independientemente de toda huella visible de cita, alusión, etc." (Charaudeau y Maingueneau, 2005, p. 299).

4. En este y en los demás ejemplos que presentaremos, el contenido citado aparecerá entre los signos "<" “>” y especificaremos entre paréntesis, al final de cada caso, si se trata de ED, EI o CO.

5. Los códigos de las entrevistas deben leerse de la siguiente manera: la primera parte de dicho código contiene las partículas fran, ignac, león, peny, rom o sand, que hacen referencia al colegio donde se realizó 
Por su parte, el EI implica reproducir las palabras adaptándolas al contexto de enunciación del sujeto que cita. Se caracteriza por presentar la voz del narrador; en estos casos, "se reproducen las palabras pronunciadas adaptándolas al sistema de referencia deíctica del hablante" (RAE, 2009, p. 3274). Este tipo de cita suele estar formado por un verbo de comunicación y una oración subordinada sustantiva encabezada, casi siempre, por la conjunción que, como en (2) o, en menor medida, por si.

(2) *CHI: pa' ir \# [c] le dije a mi abuelo [c] que yo iba iba pa' la calle [c] (EI) (Leonwilson)

La CO es un caso particular de DR porque se trata de la inclusión de un elemento paralingüístico (un sonido sin significado léxico) dentro del relato conversacional. Aunque Shiro (2012) incluye las onomatopeyas como un tipo de cita directa, las hemos considerado aparte, es decir, como citas de paralenguaje. Estos sonidos tienen especial importancia por la forma en que los nińos hacen uso de ellos. Recordemos que aún están en el proceso de desarrollar un léxico más o menos estable. Los hablantes introducen en su relato onomatopeyas que hacen referencia a una acción o hecho importante dentro de su narración. El emisor del mensaje emplea estos sonidos porque los considera importantes en su relato; como recurso vivificador sirven para representar — por aproximación — un hecho paralingüístico, como en (3):

(3) *CHI: y entonces dio la vuelta así en aire [c], <gua> ["], y se cayó, <pun> [“] [c] (CO) (Franewar)

\section{Estudios previos}

En los últimos 25 años, diversos autores se han encargado de dar cuenta del discurso referido en el habla infantil desde diferentes perspectivas (Hickmann, 1982 y 1993; Lucariello y Nelson, 1987; Goodwin, 1990; Özyürek, 1996; Prego Vázquez, 2005; entre otros). Más recientemente, la recolección de diversos corpus de habla, sobre todo en español, ha hecho posible que los investigadores exploren el uso del DR en este tipo de textos. En lo que respecta al habla infantil, destacan los estudios de Mahler (1997), Shiro (2007) y (2012), y Erbolato (2011). Aunque no nos detendremos en la descripción de los resultados de cada estudio, ${ }^{6}$ es importante resaltar algunos aspectos puntuales relacionados con nuestro análisis.

\footnotetext{
la entrevista (fran=Franciscano; ignac=San Ignacio; leon=Leoncio Martínez; peny=El Peńón; rom=Rómulo Gallegos; sand=Santiago de León); luego de la partícula se especifica el nombre del hablante. Por ejemplo: leonmailín (leon=colegio Leoncio Martínez/ mailín=nombre de la niña, Mailín).

6. Para una descripción detallada de los resultados de estas investigaciones, véase Pinto (2014).
} 
Por ejemplo, Mahler (1997) encuentra, curiosamente, que en su muestra de 15 niños la mayoría utiliza el EI para reproducir lo dicho por los personajes de una historia. En el caso de Shiro (2007), la autora identifica, a partir de un corpus de 113 nińos, cuatro tipos de cita: ED, estilo de reporte libre, ${ }^{7}$ EI y CO. De estos cuatro tipos, los más usados son el ED y la CO. En la investigación llevada a cabo por la misma autora en 2012, con el mismo corpus anterior, pero con una muestra de 107 niños, Shiro comprobó una vez más que la cita tradicional (sobre todo en ED) y las $\mathrm{CO}$ contribuyen a la dramatización de las narraciones.

Por último, Erbolato (2011) hace una propuesta de análisis para estudiar la interacción de códigos verbales y no verbales en el habla infantil, para lo cual utiliza un corpus compuesto por un grupo de muestras orales provenientes de nińos en edad escolar (de 5, 8 y 10 años) nativos de São Paulo. Como resultado general, Erbolato concluye que el funcionamiento de los códigos verbales y no verbales son elementos difíciles de separar en la interacción, sobre todo al momento de narrar y representar - y más aún si se trata de discurso infantil- porque los elementos no verbales, complementados con los verbales, pueden agregar dramatismo e información complementaria a la actividad narrativa. Cuando el narrador imprime elementos de teatralización (teatralização) al hecho narrado recurre, también, a gestos comunicativos o elementos paralingüísticos para mantener la sincronía comunicacional entre hechos y palabras (Erbolato, 2011, p. 110).

\section{Método}

La muestra analizada (Tabla 1) forma parte del Corpus de habla infantil recogido por Martha Shiro en $1996^{8}$ y consta de 28 entrevistas hechas a 14 niñas y 14 niños, con edades comprendidas entre los 6,5 y los 10,9 años de edad. ${ }^{9}$ Atendiendo a criterios de

7. El reporte libre, como lo denomina la autora, se refiere a los casos en los que se mantiene la intención citativa, pero no se incluye el contenido exacto de la cita, como en: "Entonces empezamos a jugar la ouija con una amiguita de nosotros nos cuenta una cosa que a su hermana le pasó jugando la ouija" (énfasis añadido) (Shiro, 2007, p. 73).

8. Este corpus consta de 111 entrevistas a niños estudiantes de primaria pertenecientes a seis diferentes colegios de Caracas. Las instituciones educativas seleccionadas sirven de contexto para inferir el estrato socioeconómico al que pertenecen los hablantes, pues las diferencias entre el contexto socioeconómico de los niños de colegios públicos y los de colegios privados son muy marcadas. En total, se trata de 51 niñas y 60 nińos que debieron realizar cuatro tareas narrativas, es decir, debieron elaborar cuatro relatos coherentes con el andamiaje propuesto por Shiro. En dos de las tareas narrativas debían contar hechos reales (una historia en la que hubieran sentido miedo y una en la que los hubieran llevado al médico); y en las otras dos, hechos ficticios (narrar una película o episodio de su programa favorito y narrar una película muda que cada hablante vio antes de la entrevista).

9. En un estudio posterior analizaremos la influencia de las variables sociales edad, sexo-género y nivel socioeconómico de los niños de la muestra. Aquí, por cuestiones de espacio, no nos detendremos en este aspecto. 
afijación uniforme, las entrevistas fueron elegidas con el fin de que hubiera un continuum en la edad de los hablantes y de que dichas edades se repitieran en ambos sexos, es decir, debía haber un niño y una niña de 6,5 años, de 7,6, de 9,6, y así sucesivamente hasta llegar a 10 ańos y 9 meses de edad (v. Tabla 1).

TABla I: LA MUESTRA DE ESTUdio

\begin{tabular}{|l|l|l|l|}
\hline \multicolumn{1}{|c|}{ Edad/Sexo } & \multicolumn{1}{c|}{ Niños } & \multicolumn{1}{c|}{ Niñas } & \multicolumn{1}{c|}{ Total } \\
\hline Grupo etario A (de 6,5 a 7,8 años) & 7 & 7 & 14 \\
\hline Grupo etario B (de 9,6 a 10,9 años) & 7 & 7 & 14 \\
\hline Total & 14 & 14 & 28 \\
\hline
\end{tabular}

Tomamos como unidad de análisis el enunciado por ser un concepto de la pragmática más apegado a las estructuras encontradas en la muestra. Como es bien sabido, el enunciado está vinculado a un contexto y posee significado dependiendo de la comprensión y la interpretación. Puede ser una oración completa o simplemente una frase (Frías Conde, 2001, pp. 4-5).

En términos sintácticos, la cita puede estar compuesta por una expresión introductora - que sirve para incluir la voz ajena dentro del discurso propio- y un enunciado o cláusula reportada, según sea el caso (Maldonado, 1999). En el corpus objeto de estudio, el final de cada cláusula aparece marcado con el signo [c] en todos los turnos de habla de los niños, y con el signo ["], aquellos segmentos donde hay contenido citado.

En esta investigación analizamos aquellas estructuras formadas por una expresión introductora o cláusula de reporte y una cláusula reportada. La unión de estos dos elementos incluye, por lo general, el CC en ED, como en (1); en EI, como en (2); u onomatopeyas, como en el ejemplo (3). También tomamos en cuenta aquellas citas que carecían de marco introductor, pero que sí tenían CC, como (4):

(4) *CHI: <mamá, voy a ir a jugar con una amiguita> [“] [c] (ED) (Santdaniela)

En el análisis de los casos seguimos el modelo propuesto por Gallucci (2009) para el estudio de la citas y, también, a Shiro (2007). En investigaciones posteriores sobre el tema, este modelo se ha ido afinando y se ha aplicado al estudio de diversos corpus de habla adulta del español venezolano (Gallucci 2012a, 2012b, 2013, 2014; Gallucci y Vargas 2015). En esta oportunidad, consideramos cuatro aspectos. A excepción del tipo de narración, las categorías objeto de estudio se recogen tanto en el modelo original como en los distintos estudios que acabamos de referir. En primer lugar, el tipo de cita (ED, 
EI, CO). En segundo lugar, distinguimos los marcos introductores de estas, como en (5), donde se emplea el marcador discursivo entonces; y, en el caso de los verbos, los más frecuentes empleados por los niños para tal fin, como decir (6), que es de comunicación, u otros, como buscar (7), que forma parte de los verbos de percepción.

(5) ${ }^{*} \mathrm{CHI}$ : entonces <usa la patineta> ["] [c] (ED) (Penyeduardo)

(6) *CHI: y entonces cuando me dijo [c] que me desmayé ["] [c] este \# me pusieron la cosa esa [c] pa’ escuchar en la barriga, así [c] (EI) (Romyanis)

(7) *CHI: y lo buscan <eeh, eeh> [“] [c] (CO) (Penyeduardo)

Otros tipos de estructuras observadas en la muestra fueron $(y)+q u e(8)$, sintagmas nominales (9) o bien cláusulas reportadas introducidas sin ninguna de las opciones anteriores — citas sin marco—, que hemos distinguido con $\varnothing$, como en (10).

(8) *CHI: y el chamito $y$ que <ay, no quiero [c] jugar> [“] [c] (ED) (Penycesar)

(9) *CHI: entonces todo el mundo <león> ["] [c] (ED) (Penystefany)

(10) ${ }^{*} \mathrm{CHI}$ : entonces ella se que \# los padres se quedaron asi ${ }^{10}[\mathrm{c}]$ viéndola [c] $\varnothing<$ para \# para dónde vas> ["]?[c] (ED) (Ignacsara)

En tercer lugar, tomamos en cuenta la atribución de la palabra, esto es, ver en qué medida están presentes la autocita — palabras propias o del propio grupo- (11) y la heterocita - palabras de los otros- (12) en el discurso reproducido por los niños (Marcuschi, 1997).

(11) *CHI: y yo <qué> ["] [c]? (ED) (Santmaría)

(12) *CHI: y entonces mi mamá me dijo [c] <no, sólo que te desmayaste, Yanis> ["] [c] (ED) (Romyanis)

Por último, en cada caso de DR observamos si formaba parte de una narración real (perteneciente a relatos en los que el nińo cuenta un hecho real o una anécdota personal), como en (13); o ficticia (aquella relativa a historias fantásticas, programas de televisión o alguna película que el niño hubiera visto), como en (14).

(13) ${ }^{*} \mathrm{CHI}$ : entonces de repente uno dijo [c] <hay una culebra> [“] [c] (ED) (Ignacrodrigo)

\footnotetext{
10. Aunque no contamos con registro audiovisual del corpus, es muy probable que en este caso la cita en ED esté acompañada, además, por una cita de gestos encabezada por así, es decir, que el niño imite la mirada de los padres que refiere en el relato. Para más información sobre las citas gestuales en la oralidad, consúltese Camargo (2004).
} 
(14) *CHI: entonces le dijo a la \# al padre [c] que a lo mejor se había caído en la carretera [c] (EI) (Penystefany) ${ }^{11}$

De cada entrevista seleccionamos los enunciados de DR, con o sin marco, siempre que se tratase de casos de ED o EI, o bien que fueran una CO. Siguiendo la propuesta de Gallucci (2009), excluimos: i) enunciados inteligibles, ii) casos en que el verbo de comunicación funciona como marcador discursivo y no como introductor de cita; iii) cuando los verbos tienen valor realizativo, (en primera persona del presente y supongan la realización de una acción); iv) cuando decir tiene función o significado de 'apodar' o 'llamar', y v) cuando el hablante narra un suceso de habla sin especificar el mensaje transmitido.

\section{Análisis y resultados}

\subsection{Estilo directo, estilo indirecto y cita onomatopéyica}

En la muestra encontramos un total de 343 citas cuya distribución ofrecemos en la Tabla 2 .

TABLA 2: ED, EI y CO

\begin{tabular}{|l|l|l|}
\hline \multicolumn{1}{|c|}{ Estilo } & No de casos & \multicolumn{1}{c|}{$\%$} \\
\hline ED & 205 & 59,8 \\
\hline CO & 119 & 34,6 \\
\hline EI & 19 & 5,6 \\
\hline Total & 343 & 100 \\
\hline
\end{tabular}

Como puede apreciarse en dicha tabla, el uso del ED es mucho más frecuente en el habla infantil que el EI, como suele ocurrir también con los adulto. De un total de 343 casos, el ED se emplea 205 veces, lo que representa el 59,8\% del total. Le sigue en frecuencia la CO con 119 registros $(34,6 \%)$ y el EI con 19 casos que representan tan solo el $5,6 \%$ de 343 .

La investigación de Shiro (2007) arrojó resultados similares, lo que la lleva a afirmar que la preferencia de los hablantes por el ED puede deberse a que este tipo de cita le

11. Stefany cuenta un fragmento de la película Picnic que vio como parte de las tareas propuestas por Shiro (1996) en el desarrollo de las entrevistas. 
permite al niño —que apenas está desarrollando su habilidad discursiva - representar más fácilmente los cambios de turnos de habla dentro de su propio discurso. Es importante señalar, además, que entre los 6 y 10 años de edad los niños suelen apelar a las herramientas discursivas que les sirvan para expresar lo que quieren decir de modo más práctico y con menor esfuerzo cognitivo. Mientras que con la CO ocurre prácticamente lo mismo que con el ED, el EI, en cambio, supone cambios deícticos importantes en la estructura de la frase reproducida.

A la par de lo anterior, la frecuencia de uso de la CO puede estar dada por el hecho de que los hablantes aún están en el proceso de integrar nuevas palabras a su vocabulario y, como comenta Shiro (2012), el uso de onomatopeyas contribuye a la dramatización de las narraciones, lo que a su vez coadyuva a mantener la atención del interlocutor. Sin embargo, todos los elementos paralingüísticos que el hablante pueda agregar a su discurso dependerán, también, de su conocimiento del mundo, de su capacidad memorística y de la relación que guarden dichos elementos con los hechos que el niño refiera en su discurso, tal como apunta Erbolato (2011).

\subsection{Marcos introductores de DR}

Como hemos señalado anteriormente, existen varias formas de introducir el DR en la oralidad. Si bien es cierto que en el discurso escrito es casi indispensable usar un verbo de comunicación, en el discurso oral esto no es obligatorio, pues los hablantes se valen de otro tipo de construcciones para reproducir palabras. En la Tabla 3 puede observarse la distribución de cada marco introductor que encontramos en la muestra analizada.

Tabla 3: Marcos introductores

\begin{tabular}{|l|l|l|l|l|l|}
\hline \multicolumn{2}{|c|}{ Marcos introductores } & \multicolumn{1}{c|}{ ED } & \multicolumn{1}{|c|}{ EI } & \multicolumn{1}{c|}{ CO } & \multicolumn{1}{c|}{ Total } \\
\hline \multirow{2}{*}{ Verbo } & $\mathrm{N}$ & 78 & 19 & 43 & 140 \\
\cline { 2 - 6 } & $\%$ & 22,8 & 5,6 & 12,5 & 40,9 \\
\hline \multirow{3}{*}{$\varnothing$ verbo } & $\mathrm{N}$ & 82 & 0 & 45 & 127 \\
\cline { 2 - 6 } & $\%$ & 23,9 & 0 & 13,1 & 37 \\
\hline \multirow{3}{*}{$y+$ que } & $\mathrm{N}$ & 24 & 0 & 14 & 38 \\
\cline { 2 - 6 } & $\%$ & 7 & 0 & 4,1 & 11,1 \\
\hline \multirow{2}{*}{$y+$ SN } & $\mathrm{N}$ & 15 & 0 & 6 & 21 \\
\cline { 2 - 6 } & $\%$ & 4,4 & 0 & 1,7 & 6,1 \\
\hline
\end{tabular}




\begin{tabular}{|l|l|l|l|l|l|}
\hline \multirow{2}{*}{$y+$ marcador } & $\mathrm{N}$ & 5 & 0 & 6 & 11 \\
\cline { 2 - 6 } & $\%$ & 1,4 & 0 & 1,7 & 3,1 \\
\hline \multirow{3}{*}{ Adverbio asi } & $\mathrm{N}$ & 1 & 0 & 5 & 6 \\
\cline { 2 - 6 } & $\%$ & 0,3 & 0 & 1,5 & 1,8 \\
\hline \multirow{3}{*}{ Subtotal } & $\mathrm{N}$ & 205 & 19 & 119 & 343 \\
\cline { 2 - 6 } & $\%$ & 59,8 & 5,6 & 34,6 & 100 \\
\hline
\end{tabular}

Como se aprecia en la Tabla 3, la mayoría de las citas son introducidas por verbos (140 casos, para un 40,9\% de la muestra). Estos casos son seguidos, en orden decreciente, por aquellos introducidos $\varnothing$ verbo (127 casos, para un 37\% de la muestra). Las demás construcciones introductoras constituyen un porcentaje mucho menor de la muestra que, sumado, alcanza tan solo el 22,1\% del total.

Como era de esperar, todos los casos de EI son introducidos por verbos. El ED, en cambio, sí se utiliza con todos los marcos, pero los casos más numerosos son los que corresponden a citas con y sin verbo, entre los cuales la diferencia de uso es muy pequeña (22,8\% de ED con verbo vs. $23,9 \%$ de ED sin él).

\subsubsection{Verbos}

Como señalamos anteriormente, el 40,9\% de las citas encontradas son introducidas con verbos, pero ¿qué clase de verbos? Los niños de la muestra, como los adultos, se inclinan por el verbo decir para insertar voces ajenas - o propias - en su discurso, lo que coincide con investigaciones previas sobre el habla infantil, como las de Shiro (2007) y (2012). Encontramos también DR con los verbos hacer y empezar, como en (15) y (16), respectivamente.

(15) *CHI: y la culebra me hizo <pun> ["] [c] (CO) (Ignacrodrigo)

(16) *CHI: y entonces la mamá se pone brava [c] y \# y entonces empieza <aaah> ["] [c] (CO) (Romdaniel)

Agrupamos en la categoría "otros" aquellos verbos cuya frecuencia de aparición no superó los 4 casos (buscar, caer, correr, dar, estar, ir, lanzar, oir, pensar, salir, saltar, sonar, subir y tocar). En este interesante grupo encontramos casos como los ilustrados en (17) y (18). 
(17) *CHI: y sonaron \# [c] <antes que suenen las campanas> ["] [c] (ED) (Franmariael)

(18) *CHI: pero a mí me dio risa [c] porque el \# el esposo estaba \# porque las dos estaban [c] <me duele, me duele> ["] (ED) (Ignaccarla)

En la Tabla 4 especificamos qué verbos emplearon los niños y, en cada caso, las frecuencias absolutas y relativas encontradas.

TABla 4: Verbos introductores

\begin{tabular}{|c|c|c|c|c|c|}
\hline \multicolumn{2}{|c|}{ Verbos } & ED & EI & $\mathrm{CO}$ & Total \\
\hline \multirow[t]{2}{*}{ decir } & $\mathrm{N}$ & 67 & 19 & 2 & 88 \\
\hline & $\%$ & 47,8 & 13,6 & 1,4 & 62,8 \\
\hline \multirow[t]{2}{*}{ hacer } & $\mathrm{N}$ & 0 & 0 & 15 & 15 \\
\hline & $\%$ & 0 & 0 & 10,7 & 10,7 \\
\hline \multirow[t]{2}{*}{ empezar } & $\mathrm{N}$ & 2 & 0 & 4 & 6 \\
\hline & $\%$ & 1,4 & 0 & 2,9 & 4,3 \\
\hline \multirow[t]{2}{*}{ llamar } & $\mathrm{N}$ & 2 & 0 & 4 & 6 \\
\hline & $\%$ & 1,4 & 0 & 2,9 & 4,3 \\
\hline \multirow[t]{2}{*}{ gritar } & $\mathrm{N}$ & 3 & 0 & 2 & 5 \\
\hline & $\%$ & 2,1 & 0 & 1,4 & 3,5 \\
\hline \multirow[t]{2}{*}{ Otros } & $\mathrm{N}$ & 4 & 0 & 16 & 20 \\
\hline & $\%$ & 3 & 0 & 11,4 & 14,4 \\
\hline \multirow[t]{2}{*}{ Subtotal } & $\mathrm{N}$ & 78 & 19 & 43 & 140 \\
\hline & $\%$ & 55,7 & 13,6 & 30,7 & 100 \\
\hline
\end{tabular}

Como puede apreciarse, en la mayoría de los casos se trata del verbo de comunicación decir que aparece 88 veces, para un 62,8\% del total. Era de esperar, como ya hemos señalado, que los casos de EI se introduzcan con un verbo; llama la atención, no obstante, que en todos esos casos sea decir, y no otro verbo de comunicación, el empleado como marco. Sobre este particular, Benavent Payá (2003, p. 18) afirma que el uso de decir en presente histórico - tiempo verbal más utilizado también en nuestra muestrarepresenta "una forma estratégica del hablante para no comprometerse con el tipo de afirmación que en su día hizo el hablante citado". 
Es necesario subrayar igualmente el hecho de que el 12,5\% de los casos de CO se introducen con verbos, principalmente con hacer $(10,7 \%)$, resultados que coinciden con lo apuntado por Camargo (2004, p. 206) para el habla adulta.

\subsection{Atribución de la palabra}

Cuando estudiamos el DR, la preferencia de los hablantes por reproducir la palabra propia o ajena puede dar cuenta de qué tan comprometidos están con lo que citan o incluso qué tan confiable es el CC. La distribución de este aspecto en la muestra puede apreciarse en la Tabla 5.

TABla 5: Atribución de la PALABra

\begin{tabular}{|l|c|c|c|c|c|}
\hline \multicolumn{2}{|c|}{ Atribución } & ED & EI & CO & Total \\
\hline \multirow{2}{*}{ Heterocita } & $\mathrm{N}$ & 189 & 17 & 112 & 318 \\
\cline { 2 - 6 } & $\%$ & 55,1 & 5 & 32,6 & 92,7 \\
\hline \multirow{2}{*}{ Autocita } & $\mathrm{N}$ & 16 & 2 & 7 & 25 \\
\cline { 2 - 6 } & $\%$ & 4,7 & 0,6 & 2 & 7,3 \\
\hline \multirow{2}{*}{ Subtotal } & $\mathrm{N}$ & 205 & 19 & 119 & 343 \\
\cline { 2 - 6 } & $\%$ & 59,8 & 5,6 & 34,6 & 100 \\
\hline
\end{tabular}

Se trata, en total, de 318 heterocitas y 25 autocitas. En ambos tipos de atribución, la mayoría de los casos, y por mucha diferencia, corresponde a citas en ED (189 de heterocitación y 16 de autocitación, para un $55,1 \%$ y un 4,7\%, respectivamente). En lo que al EI se refiere, solamente encontramos 2 autocitas que representan apenas el 0,6\% del total, pues el resto (17 casos, para un 5\%) corresponde a heterocitas. La diferencia entre ambas formas de atribución para el EI es de 4,4\%.

Ahora bien, cuando se trata de CO, la diferencia porcentual entre autocitas y heterocitas es de $2 \%$ vs. $32,6 \%$ (esto es 7 casos de autocita contra 112 de heterocita, lo que representa una diferencia de $30,6 \%$ entre un tipo de atribución de palabra y otro). Todo esto indica que, al menos según nuestros datos, existe preferencia por reproducir sonidos emitidos por otros sujetos. En nuestra muestra, la gran cantidad de heterocitas puede deberse a que los nińos produjeron una mayor cantidad de citas en narraciones ficcionales (cuentos, películas, episodios de programas de televisión) que en 
las personales, lo que podría justificar que usen el DR para citar casi exclusivamente (en un $92,7 \%$ del total) la palabra del otro.

\subsection{Tipo de narración}

En función de las características de las entrevistas, en las que los niños debían contar una narración de experiencia personal y otra relacionada con alguna película que hayan visto, quisimos ver cómo se distribuyen las citas encontradas según este aspecto. La preferencia de los nińos de la muestra en este sentido se aprecia en la Tabla 6.

TABLA 6: Tipo DE NARRACióN

\begin{tabular}{|l|c|c|c|c|c|}
\hline \multicolumn{2}{|c|}{ Narración/Cita } & ED & EI & CO & Total \\
\hline \multirow{2}{*}{ Ficción } & N & 130 & 9 & 100 & 239 \\
\cline { 2 - 6 } & $\%$ & 37,9 & 2,7 & 29,1 & 69,7 \\
\hline \multirow{2}{*}{ Realidad } & $\mathrm{N}$ & 75 & 10 & 19 & 104 \\
\cline { 2 - 6 } & $\%$ & 21,9 & 2,9 & 5,5 & 30,3 \\
\hline \multirow{3}{*}{ Subtotal } & $\mathrm{N}$ & 205 & 19 & 119 & 343 \\
\cline { 2 - 6 } & $\%$ & 59,8 & 5,6 & 34,6 & $100 \%$ \\
\hline
\end{tabular}

De lo anterior se desprende que los nińos utilizan la cita sobre todo para contar hechos ficticios, pues la diferencia entre la narración de ficción y realidad es de $239(69,7 \%) v$ s. $104(30,3 \%)$ a favor de la primera, lo cual coincide con lo apuntado por Shiro (2012). También podemos apreciar la preferencia de la CO, indicio de imitación y teatralización, en las narraciones de ficción (100 casos frente a 19 en la narración de hechos reales); situación similar se observa con el ED (21,9\% frente a 37,9\%). Por último, en lo que respecta al EI, el número de casos (10 vs. 9) no parece indicar preferencia por un tipo de narración u otro.

\section{Conclusiones}

El análisis ha permitido observar que los niños se inclinan fundamentalmente por el uso del ED para citar y contar sus historias, seguido de la $\mathrm{CO}$; tendencia que también se observa en investigaciones anteriores sobre el tema (Shiro 2007 y 2012). Shiro (2012) sostiene que se trata de una herramienta de vivificación que contribuye a mantener 
la atención del oyente y que su uso frecuente en el habla infantil se debe a que los niños aún están desarrollando su habilidad discursiva, planteamiento que compartimos. Por su parte, Erbolato (2011) considera que las emisiones paralingüísticas dependerán primordialmente del conocimiento del mundo y de la capacidad de memorización del hablante, así como de su habilidad para conectar el sentido de las onomatopeyas con el continuum de su relato. Sería interesante observar esto con más detalle en futuras investigaciones.

De las citas encontradas, un poco más del $40 \%$ se introduce con verbos y $37 \%$ sin ellos; como hemos visto, estas dos son las formas preferidas de los hablantes. Además de los otros marcos a los que nos hemos referido en la sección correspondiente, encontramos citas introducidas con el adverbio asi, de las cuales no hay precedentes en los estudios previos reseñados, pero sí en la investigación de Camargo (2004) sobre el habla adulta. Aunque se trata apenas de 6 casos, que representan el 1,8\% de la muestra, creemos que constituyen un hallazgo importante para el tema estudiado, en virtud de que no se trata de un marco introductor prototípico en español.

Constatamos también que los hablantes se inclinaron sobre todo por el verbo decir y en presente histórico, lo que coincide con investigaciones anteriores como las de Shiro (2007) y (2012) y Benavent Payá (2003). Esta preferencia, que también se registra en el habla adulta, puede deberse al hecho de que, como es sabido, decir es el "el más usual e incoloro de los verbos que introducen estilo directo” (cf. Dámaso Alonso 1973: 196) y, por tanto, el más neutral de los verbos de comunicación. Piénsese, por ejemplo, la diferencia entre este último y los verbos afirmar, asegurar o aseverar, que implican mayor fuerza asertiva y suponen un contenido de subjetividad añadido. Por su parte, el verbo de creación hacer y el de fase empezar, aunque se emplean en menor medida, se usan sobre todo para insertar en el discurso citas onomatopéyicas, como ya ha apuntado Camargo (2004).

En relación con la atribución de las palabras citadas, el análisis arrojó una clara inclinación por la heterocita. No encontramos investigaciones previas acerca de si los niños prefieren citarse a sí mismos o a otros; sin embargo, los resultados sobre este punto son similares a los obtenidos en otras investigaciones como las de Gallucci (2013) y Fernández (2011) — con corpus de habla adulta— pero difieren de los expuestos en el trabajo de Gallucci (2009), en el que los hablantes prefirieron las autocitas. Debemos destacar que, de momento, no existe una tendencia clara en este sentido. Esto pareciera indicar que la preeminencia de la heterocita sobre la autocita $\multimap$ o viceversa— dependerá de la muestra en cuestión.

Igualmente, un hallazgo importante de este trabajo fue descubrir que, como narradores, los niños dotan a sus personajes de estados emocionales, sobre todo si se trata de seres ficticios. En las entrevistas analizadas, una vez que los niños ven una película muda y la narran, describen cómo se siente el personaje (triste, emocionado, ansioso) y suelen usar la CO para manifestar dichos estados. Sería interesante estudiar con detalle 
esto último en una investigación de mayor alcance con entrevistas tanto del español americano como del peninsular a fin de proponer una clasificación de las $\mathrm{CO}$ empleadas en la reproducción del discurso.

\section{Referencias}

Alonso, D. (1973). El anuncio del estilo directo en el Poema del Cid y en la épica francesa. En D. Alonso (Coord.), Obras completas. Estudios y ensayos sobre literatura II. Desde los origenes románicos hasta finales del siglo XVI (pp. 195-214). Madrid: Gredos.

Authier-Revuz, J. (1982). Hetérogénéité montrée et héterogénéité constitutive: élements pour une approche de l'autre dans le discours. DRLAV: Revue de linguistique, 26, 91151.

Authier-Revuz, J. (1984). Heterogéneité(s) énonciative(s). Langages, 73, 98-111.

Authier-Revuz, J. (1995). Ces mots qui ne vont pas de soi: Boucle réflexive e non coïncidence du dire. París: Larousse.

Authier-Revuz, J. (1996). Remarques sur la catégorie de «l'îlot textuel». Cahiers du Français Contemporain, 3, 91-115.

Bajtín, M. (1981). The dialogic imagination. Austin: University of Texas Press.

Bajtín, M. (1982). Estética de la creación verbal. México: Siglo XXI editores.

Bolívar, A. (1998-1999). El reporte de la experiencia con decir en el habla de Caracas. Homenaje a Ambrosio Rabanales. Boletin de Filología de la Universidad de Chile, XXXVII, 181-206.

Benavent Payá, E. (2003). ¿Por qué contamos nuestras historias cotidianas en estilo directo? Foro Hispánico, 23, 11-20.

Camargo, L. (2004). La representación del discurso en la narración oral conversacional. Estudio sociopragmático (Tesis doctoral). Universidad de Alcalá, España.

Charaudeau, P., y D. Maingueneau (Eds.). (2005). Diccionario de análisis del discurso. Buenos Aires: Amorrortu.

Ducrot, O. (1986). El decir y lo dicho. Polifonía de la enunciación. Barcelona: Paidós.

Erbolato, L. (2011). Aspectos lingüísticos e discursivos em narrativa oral infantil: Algunas reflexôes. Lingüistica, 25, 92-110.

Fernández, M. (2011). Uso del discurso directo e indirecto en el habla de Mérida (Trabajo de fin de grado). Universidad de Los Andes.

Frías Conde, X. (2001). Introducción a la pragmática. Recuperado de http://www. romaniaminor.net/ianua/sup/sup05.pdf

Gallucci, M. (2009). Nos fuimos a la casa y mi mamá: “¿Estaban lanzándose por la quebrada?”. “¡No, mamá!", "Claro que sî”. Estudio piloto de las citas conversacionales en el habla de Caracas. Núcleo, 26, 75-98.

Gallucci, M. (2012a). Sintaxis de las citas en estilo directo e indirecto con verbo en el habla caraqueña. Lingüistica, 28, 223-246. 
Gallucci, M. (2012b). Yo digo: "Bueno, vale, de Venezuela, Caracas, y del interior, Petare": Las citas en estilo directo e indirecto en el español hablado en Caracas. Núcleo, 29, 11-37.

Gallucci, M. (2013). Más sobre el estilo directo e indirecto en el español de Caracas. Lengua y Habla, 17, 89-111.

Gallucci, M. (2014). Entonces un muchacho llega y me dice: "Mira, regáleme un bolívar". Las citas en estilo directo e indirecto en el Corpus diacrónico del habla de Caracas 1987/2013. Boletín de Lingüistica, XXVI(41-42), 43-72.

Gallucci, M. y Vargas, K. (2015). Estilo directo e indirecto en el Corpus sociolingüístico de Caracas 1987. Anuario de Letras. Lingüistica y Filologia, III(2), 65-103.

Goodwin, M. (1990). He-Said-She Said: Talk as Social Organization among Black Children. Bloomington: Indiana University Press.

Hockett, C. (1958). Curso de lingüistica moderna. Buenos Aires: Eudeba.

Hickmann, M. (1982). Metapragmatics in child language. En E. Mertz y R. Parmentier (Eds.), Semiotic mediation: psychological and sociocultural perspectives (pp. 177-201). Orlando, FL: Academic Press.

Hickmann, M. (1993). The boundaries of reported speech in narrative discourse: some developmental aspects. En A. Lucy (Ed.), Reflexive language: reported speech and metapragmatics (pp. 63-90). Cambridge: Cambridge University Press.

Jakobson, R. (1975). Ensayos de lingüistica general. Barcelona: Seix Barral.

Lucariello, J., y Nelson, K. (1987). Remember and planning talk between mothers and children. Discourse Processes, 10, 457-472.

Lucy, J. (Ed.). (1993). Reflexive language. Reported speech and metapragmatics. Cambridge: Cambridge University Press.

Mahler, P. (1997). Discurso referido y perspectiva narrativa en narraciones orales infantiles. Lenguas modernas, 24, 61-82.

Maldonado, C. (1999). Discurso directo y discurso indirecto. En I. Bosque y V. Demonte (Eds.), Gramática descriptiva de la lengua española (vol. 3, pp. 3551-3595). Madrid: Espasa-Calpe.

Marcuschi, L. (1997). Citação de fala na interacäo verbal como fala idealizada. En A. Bolívar y P. Bentivoglio (Eds.), Actas del I Coloquio Latinoamericano de Analistas del Discurso (pp. 187-202). Caracas: Universidad Central de Venezuela.

Martinet, A. (1972). Elementos de lingüistica general. Madrid: Gredos.

Özyürek, A. (1996). How children talk about a conversation. Journal of Child Language, 23(3), 693-714.

Pinto, Y. (2014). Yo te digo. Tú me dices. Aproximación al uso del reporte oral en el discurso infantil (Trabajo de fin de grado). Universidad Central de Venezuela, Venezuela.

Prego Vázquez, G. (2005). Recursos gramaticales y dinámicas interaccionales de la protofunción discursiva 'cita' en el habla infantil. En E. Serra y M. Veyrat (Eds.), Estudios de Lingüistica Clínica. Problemas de eficacia comunicativa. Descripción, 
detección, rehabilitación (vol. 4, pp. 139-159). Valencia: Universitat de València / AVaLCC.

Real Academia Española y Asociación de Academias de la Lengua Española. (2009). Nueva gramática de la lengua española. Tomo II. Madrid: Espasa.

Reyes, G. (1995). Los procedimientos de cita: estilo directo y estilo indirecto. Madrid: Arco/ Libros.

Rosier, L. (1999). Le discours rapporté. Historie, théories, pratiques. Bruselas: Duculot.

Shiro, M. (1996). CHILDES database. Romance languages corpora, Spanish-Shiro. Disponible en http://childes.psypsy.cmu.edu/data/Romance/ Spanish/

Shiro, M. (2007). La construcción del punto de vista de los relatos orales de niños en edad escolar. Un análisis discursivo de la oralidad. Caracas: Fondo Editorial de Humanidades, Universidad Central de Venezuela.

Shiro, M. (2012). Y entonces le dijo... La representación del habla en las narraciones de niños venezolanos. Caracas: Universidad Central de Venezuela. 